\title{
Efficient Near-ML Detection for MIMO Channels: The Sphere-Projection Algorithm
}

\author{
Dominik Seethaler, Harold Artés, and Franz Hlawatsch \\ Institute of Communications and Radio-Frequency Engineering, Vienna University of Technology \\ Gusshausstrasse 25/389, A-1040 Vienna, Austria \\ phone: +43 158801 38958, fax: +43 158801 38999, email: dominik.seethaler@tuwien.ac.at \\ web: http://www.nt.tuwien.ac.at/dspgroup/time.html
}

\begin{abstract}
It is well known that suboptimal detection schemes for MIMO spatial multiplexing systems (equalization-based as well as nulling-and-cancelling detectors) cannot exploit all of the available diversity. In this paper, we show that this inferior performance is primarily caused by poorly conditioned channel realizations. We then present the novel sphere-projection algorithm (SPA) that is robust to poorly conditioned channels. The SPA is a computationally efficient add-on to standard suboptimal detectors. Simulation results show that the SPA is able to achieve near-ML performance and significantly increased diversity gains. The SPA's computational complexity is comparable to that of nulling-and-cancelling detectors and only a fraction of that of the Fincke-Phost sphere-decoding algorithm for ML detection.
\end{abstract}

\section{INTRODUCTION}

It is well known that the diversity offered by multipleinput/multiple-output (MIMO) fading channels cannot be fully exploited by suboptimal detectors (linear equalization and nulling-and-cancelling schemes [2]). Maximum-likelihood (ML) detection exploits all of the available diversity but tends to be computationally intensive. Thus, there is a strong demand for computationally efficient suboptimal detectors that can exploit a large part of the available diversity.

In this paper, we present the novel sphere-projection algorithm (SPA) for data detection in spatial multiplexing systems, and we show that the SPA can achieve near-ML performance at low complexity. The SPA is motivated by the observation that the inferior average performance of standard suboptimal detectors is mainly caused by the occurrence of "bad" (i.e., poorly conditioned) channel realizations. The SPA is a simple add-on to standard suboptimal schemes that significantly increases the robustness of these schemes to bad channels.

Our paper is organized as follows. In the remainder of this section, we describe the system model and briefly review existing detection schemes. In Section II, the effect of bad channels on equalization-based detection is analyzed. The SPA is introduced in Section III. Finally, in Section IV the performance of the SPA is assessed through simulation results.

\section{A. System Model}

We consider a MIMO channel with $M_{\mathrm{T}}$ transmit antennas and $M_{\mathrm{R}} \geq M_{\mathrm{T}}$ receive antennas (briefly termed an $\left(M_{\mathrm{T}}, M_{\mathrm{R}}\right)$

Funding by FWF grant P15156-N02. A more detailed presentation of this work can be found in [1]. channel). We assume a spatial multiplexing system such as V-BLAST [2] where the $m$ th data symbol (or layer) $d_{m}$ is directly transmitted on the $m$ th transmit antenna. For any given time instant, this leads to the well-known baseband model

$$
\mathbf{r}=\mathbf{H d}+\mathbf{w},
$$

with the data vector $\mathbf{d} \triangleq\left(d_{1} \cdots d_{M_{\mathrm{T}}}\right)^{T}$, the $M_{\mathrm{R}} \times M_{\mathrm{T}}$ channel matrix $\mathbf{H}$, the received vector $\mathbf{r} \triangleq\left(r_{1} \cdots r_{M_{\mathrm{R}}}\right)^{T}$, and the noise vector $\mathbf{w} \triangleq\left(w_{1} \cdots w_{M_{\mathrm{R}}}\right)^{T}$. The data symbols $d_{m}$ are assumed zero-mean and white with variance $\sigma_{d}^{2}$. The components of the noise vector $\mathbf{w}$ are assumed to be independent and circularly symmetric complex Gaussian with variance $\sigma_{w}^{2}$. The channel $\mathbf{H}$ is considered constant over a block of $N$ consecutive time instants and perfectly known at the receiver.

\section{B. Review of Detection Schemes}

Major suboptimal detection schemes for spatial multiplexing systems include linear equalization followed by quantization and nulling-and-cancelling (or decision-feedback) detection [2]. In linear equalization based schemes, the detected data vector is $\hat{\mathbf{d}}=Q\{\mathbf{y}\}$ with $\mathbf{y}=\mathbf{G r}$, where $Q\{\cdot\}$ denotes component-wise quantization according to the symbol alphabet. The zero-forcing (ZF) equalizer is given by $\mathbf{G}=$ $\mathbf{H}^{\#}=\left(\mathbf{H}^{H} \mathbf{H}\right)^{-1} \mathbf{H}^{H}$ (assuming that $\mathbf{H}$ has full rank), while the minimum mean-square error (MMSE) equalizer is given by $\mathbf{G}=\left(\mathbf{H}^{H} \mathbf{H}+\frac{\sigma_{w}^{2}}{\sigma_{d}^{2}} \mathbf{I}\right)^{-1} \mathbf{H}^{H}$ [3]. In contrast to linear equalization schemes, where all layers are detected jointly, nulling and cancelling (NC) uses a serial decision-feedback approach to detect each layer separately. NC is based on the ZF or MMSE approach [2]; the corresponding detectors will be referred to as NC-ZF and NC-MMSE, respectively.

Finally, ML detection [4], [5] yields minimum error probability for equally likely data vectors. For our system model (1) and our assumptions, the ML detector is given by

$$
\hat{\mathbf{d}}_{\mathrm{ML}}=\underset{\mathbf{d} \in \mathcal{D}}{\operatorname{argmin}}\left\{\|\mathbf{r}-\mathbf{H d}\|^{2}\right\},
$$

with $\mathcal{D}$ the set of data vectors. The computational complexity of ML detection grows exponentially with $M_{\mathrm{T}}$. Using the Fincke-Phost sphere-decoding algorithm for ML detection [4], an average complexity of roughly $\mathcal{O}\left(M_{\mathrm{T}}^{3}\right)$ is obtained [6]. 


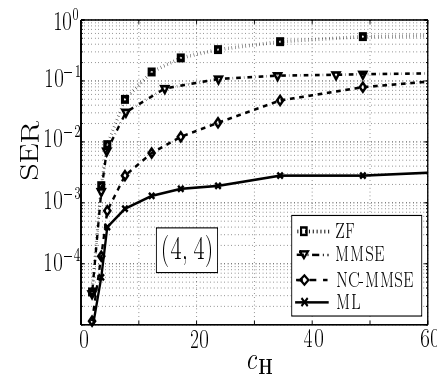

(a)

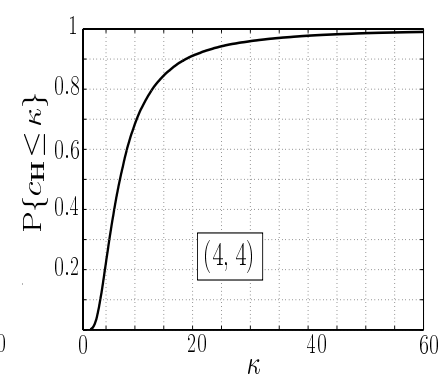

(b)
Fig. 1. Detector performance and channel condition number: (a) SER performance of various detection schemes versus the condition number $c_{\mathbf{H}}$ of the respective channel realization $\mathbf{H}$ for a $(4,4)$ MIMO channel, 4-QAM modulation, and a fixed SNR of $15 \mathrm{~dB}$, (b) cumulative distribution function of $c_{\mathbf{H}} . \mathbf{H}$ is modeled iid Gaussian.

\section{The "Bad Channel" EfFect}

As a motivation for the SPA, we first study the effect of bad channel realizations on equalization-based detection. We will use the singular value decomposition (SVD) $\mathbf{H}=\mathbf{U} \boldsymbol{\Sigma} \mathbf{V}^{H}$, where the diagonal matrix $\boldsymbol{\Sigma}$ contains the singular values $\sigma_{m}$ of $\mathbf{H}$ (assumed indexed in nonincreasing order) and the columns of $\mathbf{U}$ and $\mathbf{V}$ are the left and right singular vectors of $\mathbf{H}$, respectively [7]. The condition number $c_{\mathbf{H}}=\sigma_{1} / \sigma_{M_{\mathrm{T}}}$ is the ratio of the largest and smallest singular values; it is large for a "bad" channel.

\section{A. Detector Performance and Bad Channels}

Experiments suggest that the performance of suboptimal detection for a given channel realization $\mathbf{H}$ strongly depends on the condition number $c_{\mathbf{H}}$. In Fig. 1(a), we show the symbol error rate (SER) of various detection schemes versus $c_{\mathbf{H}}$. For this simulation, we used a $(4,4)$ channel with iid Gaussian channel matrix entries and a fixed SNR of $15 \mathrm{~dB}$. For $c_{\mathbf{H}}$ larger than about 20, we observe a significant performance gap between suboptimal detection and ML detection. Fig. 1(b) shows that channel realizations with $c_{\mathbf{H}}$ larger than about 20 occur frequently enough to cause a significant degradation of the average performance of suboptimal detection.

Because ML detection usually is too complex (in this context, we note that the Fincke-Phost sphere-decoding algorithm for ML detection has substantially increased complexity in the case of bad channels [4]), there is a strong demand for computationally efficient suboptimal detectors that are able to achieve near-ML performance. The SPA, to be presented in Section III, is designed to satisfy this demand.

\section{B. The Bad Channel Effect in the ZF Domain}

After ZF equalization (i.e., in the "ZF domain"), we obtain

$$
\mathbf{y}_{\mathrm{ZF}}=\mathbf{H}^{\#} \mathbf{r}=\mathbf{d}+\tilde{\mathbf{w}} .
$$

This is the undistorted data $\mathbf{d}$ corrupted by the noise $\tilde{\mathbf{w}}=$ $\mathbf{H}^{\#} \mathbf{w}$ that is correlated with covariance matrix

$$
\mathbf{R}_{\tilde{\mathbf{w}}}=\sigma_{w}^{2}\left(\mathbf{H}^{H} \mathbf{H}\right)^{-1}=\sigma_{w}^{2} \mathbf{V} \boldsymbol{\Sigma}^{-2} \mathbf{V}^{H} .
$$

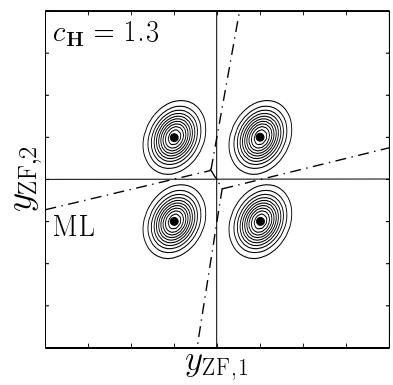

(a)

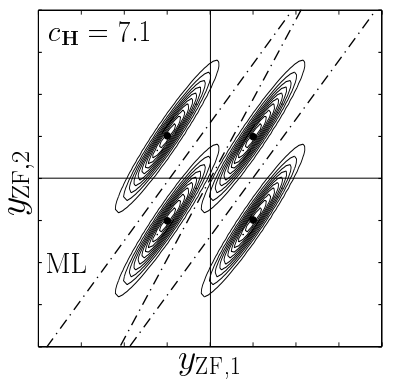

(b)
Fig. 2. Pdf contour lines and $\mathrm{ZF}$ and $\mathrm{ML}$ decision regions in the $\mathrm{ZF}$-equalized domain for a $(2,2)$ channel and BPSK modulation: (a) "Good" channel realization with $c_{\mathbf{H}}=1.3$, (b) "bad" channel realization with $c_{\mathbf{H}}=7.1$.

Hence, the contour surfaces of the probability density function (pdf) of $\tilde{\mathbf{w}}$ are hyperellipsoids whose $m$ th principal axis is given by the $m$ th eigenvector $\mathbf{v}_{m}$ of $\mathbf{R}_{\tilde{\mathbf{w}}}$ (the $m$ th column of $\mathbf{V}$ ), with its length proportional to $\sigma_{\tilde{w}, m}=\sigma_{w} / \sigma_{m}$ [8]. Thus, ZF equalization results in a distortion of the noise pdf relative to the spherical pdf geometry of w. For illustration, Fig. 2 shows the pdf of the received signal after ZF equalization for a "good" and a "bad" realization of a real-valued $(2,2)$ channel. The modulation format is BPSK. Also shown are the $\mathrm{ZF}$ decision regions (the four quadrants) and the ML decision regions (indicated by dash-dotted lines). For the good channel, the ZF and ML decision regions are similar. For the bad channel, however, they are very different; in particular, two of the boundary lines separating the ML decision regions feature an offset perpendicular to the dominant principal axis $\mathbf{v}_{M_{\mathrm{T}}}$ (which is the direction of the dominant noise component).

Also the MMSE and NC schemes are unable to properly adapt to the noise distortion caused by bad channels, even though their decision regions are somewhat better matched to the distorted pdf of $\tilde{\mathbf{w}}$ than the ZF decision regions [1].

Experiments indicate that for a bad channel, the largest ZF-domain noise component (whose variance is the largest eigenvalue $\sigma_{\tilde{w}, M_{\mathrm{T}}}^{2}$ of $\mathbf{R}_{\tilde{\mathbf{w}}}$ and whose direction is given by the principal axis $\mathbf{v}_{M_{\mathrm{T}}}$ ) tends to dominate all the other noise components. Hence, this dominant noise component causes the main part of the bad channel effects that are responsible for the poor performance of linear and NC detection.

\section{The Sphere-Projection Algorithm}

For simplicity, we hereafter assume a symbol alphabet with constant modulus $\sigma_{d}$. Here, all data vectors $\mathbf{d}$ are located on an $M_{\mathrm{T}}$-dimensional data hypersphere $\mathcal{H}$ of radius $R=$ $\sigma_{d} \sqrt{M_{\mathrm{T}}}$ about the origin. This assumption allows arbitrary PSK constellations. (Our algorithms can be extended to other symbol alphabets by using several hyperspheres.)

We also assume that the symbol alphabet is "line-structured" in that the boundaries of the symbol quantization regions in the 2-D real symbol domain are straight lines. This holds for arbitrary ASK, QAM, and PSK alphabets but not, e.g., for hexagonal constellations. Let $P$ denote the number of boundary lines. For instance, 4 -QAM has $P=2$ orthogonal 
boundary lines (see Fig. 3).

We will now describe the SPA. The SPA is an add-on to an arbitrary suboptimal detector, in the following sense. Recall from (2) that the ML detector would minimize $\|\mathbf{r}-\mathbf{H d}\|^{2}$ over the entire data vector set $\mathcal{D}$. Let $\hat{\mathbf{d}}_{\text {sub }} \in \mathcal{D}$ denote the result of the given suboptimal detector. This result can be expected to be reasonably good for a good channel. In order to improve the performance for bad channels, we additionally consider a suitably chosen set $\mathcal{D}_{+} \subset \mathcal{D}$ of valid data vectors $\mathbf{d}$ that are potentially better than $\hat{\mathbf{d}}_{\text {sub }}$ in the sense of smaller $\|\mathbf{r}-\mathbf{H d}\|^{2}$. We then minimize $\|\mathbf{r}-\mathbf{H d}\|^{2}$ over the search set $\mathcal{D}_{\mathrm{SP}}$ that consists of $\hat{\mathbf{d}}_{\text {sub }}$ and all data vectors in $\mathcal{D}_{+}$:

$$
\hat{\mathbf{d}}_{\mathrm{SP}} \triangleq \underset{\mathbf{d} \in \mathcal{D}_{\mathrm{SP}}}{\arg \min }\left\{\|\mathbf{r}-\mathbf{H d}\|^{2}\right\}, \quad \text { with } \mathcal{D}_{\mathrm{SP}} \triangleq\left\{\hat{\mathbf{d}}_{\text {sub }}\right\} \cup \mathcal{D}_{+} \cdot
$$

It remains to discuss the construction of $\mathcal{D}_{+}$.

\section{A. Combatting the Bad Channel Effect}

Let us assume a bad channel. As discussed in Section II$\mathrm{B}$, in the $\mathrm{ZF}$ domain there is a dominant noise component in the direction of $\mathbf{v}_{M_{\mathrm{T}}}$ (see Fig. 2(b)). The ZF-equalized vector $\mathbf{y}_{Z F}=\mathbf{H}^{\#} \mathbf{r}=\mathbf{d}+\tilde{\mathbf{w}}$ can be decomposed as $\mathbf{y}_{\mathrm{ZF}}=$ $\mathbf{y}_{\mathbf{v}_{M_{\mathrm{T}}}}+\mathbf{y}_{\mathbf{v}_{M_{\mathrm{T}}}}^{\perp}$, where $\mathbf{y}_{\mathbf{v}_{M_{\mathrm{T}}}}^{\perp}$ is orthogonal to $\mathbf{v}_{M_{\mathrm{T}}}$. We define the reference line $\mathcal{L}$ as the straight line parallel to the dominant noise axis $\mathbf{v}_{M_{\mathrm{T}}}$ whose offset from the origin is $\mathbf{y}_{\mathbf{v}_{M_{\mathrm{T}}}}^{\perp}$ :

$$
\mathcal{L}: \quad \mathbf{y}_{\text {ref }}(k) \triangleq k \mathbf{v}_{M_{\mathrm{T}}}+\mathbf{y}_{\mathbf{v}_{M_{\mathrm{T}}}}^{\perp}, \quad k \in \mathbb{C} .
$$

$\mathcal{L}$ passes through $\mathbf{y}_{\text {ZF }}=\mathbf{d}+\tilde{\mathbf{w}}$, which is obtained for $k=$ $\mathbf{v}_{M_{\mathrm{T}}}^{H} \mathbf{y}_{\mathrm{ZF}}$. Now consider the distance $\psi_{i} \triangleq\left\|\mathbf{r}-\mathbf{H d}^{(i)}\right\|$ for some $\mathbf{d}^{(i)} \in \mathcal{D}_{+}$. Since we assumed a bad channel, the dominant noise component in the direction of $\mathbf{v}_{M_{\mathrm{T}}}$ (i.e., of $\mathcal{L})$ is much larger than all other noise components. Therefore, it makes sense to exclude this dominant noise direction from our distance calculation. Furthermore, to simplify things, we will do as if all of the small noise components had equal variance. With these approximations, it can be shown [1] that $\mathbf{d}^{(i)}$ has a small distance $\psi_{i}$ if and only if it is close to $\mathcal{L}$. (In fact, if all nondominant noise components were exactly zero, $\mathcal{L}$ would pass right through the transmitted data vector $\mathbf{d}$.)

This suggests to construct $\mathcal{D}_{+}$as a set of data vectors that are close to the reference line $\mathcal{L}$. We will next present an efficient algorithm for constructing the data vectors $\mathbf{d}^{(i)} \in \mathcal{D}_{+}$ and determining their distances $\psi_{i}=\left\|\mathbf{r}-\mathbf{H d}^{(i)}\right\|$.

\section{B. Efficient Calculation of $\mathcal{D}_{+}$and $\psi_{i}^{2}$ : Case 1}

We first assume that $\mathcal{L}$ intersects the data hypersphere $\mathcal{H}$ (the opposite case will be considered in Section III-C). We here choose $\mathcal{D}_{+}$to consist of data vectors $\mathbf{d} \in \mathcal{H}$ close to the intersection set $\mathcal{L} \cap \mathcal{H}$. This intersection set is defined by the equation $\left\|\mathbf{y}_{\text {ref }}(k)\right\|^{2}=R^{2}$ or, equivalently,

$$
|k|^{2}+\left\|\mathbf{y}_{\mathbf{v}_{M_{\mathrm{T}}}}^{\perp}\right\|^{2}=R^{2}
$$

where $R$ is the radius of $\mathcal{H}$. In the complex $k$ plane, this represents an intersection circle $\mathcal{I}$ with radius $\tilde{R} \triangleq$ $\sqrt{R^{2}-\left\|\mathbf{y}_{\mathbf{v}_{M_{\mathrm{T}}}}^{\perp}\right\|^{2}}$. That is, (4) is satisfied by $k=\tilde{R} e^{j \phi}$ with

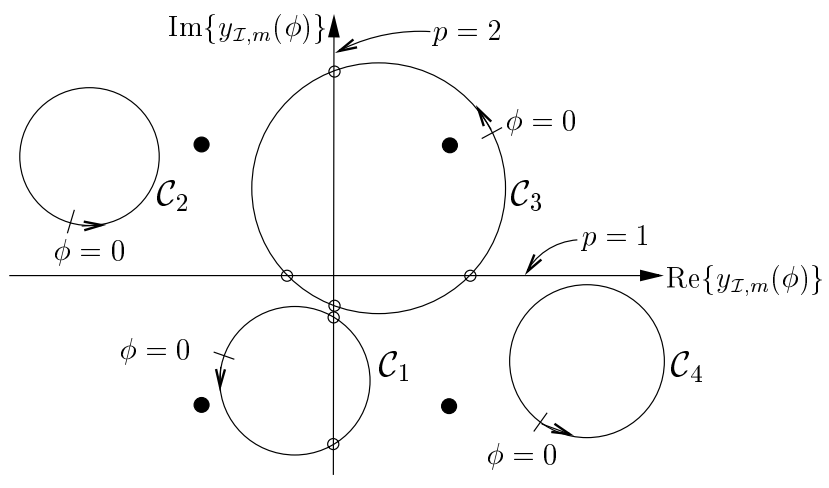

Fig. 3. Component circles $\mathcal{C}_{m}$ for a $(4,4)$ channel and 4-QAM modulation $(P=2)$. (All 4 component circles are drawn in a common complex plane.) When moving along the intersection circle $\mathcal{I}$ (by varying $\phi$ ), the $m$ th component $y_{\mathcal{I}, m}(\phi)$ of $\mathbf{y}_{\mathcal{I}}(\phi)$ moves along the component circle $\mathcal{C}_{m}$.

arbitrary angle $\phi$. The $\mathbf{y}$ vectors corresponding to $\mathcal{I}$ are thus given by $\mathbf{y}_{\mathcal{I}}(\phi) \triangleq \mathbf{y}_{\text {ref }}\left(\tilde{R} e^{j \phi}\right)=\tilde{R} e^{j \phi} \mathbf{v}_{M_{\mathrm{T}}}+\mathbf{y}_{\mathbf{v}_{M_{\mathrm{T}}}}^{\perp}$.

Evidently, the data vector nearest to $\mathbf{y}_{\mathcal{I}}(\phi)$ is obtained by component-wise quantization of $\mathbf{y}_{\mathcal{I}}(\phi)$. Thus, we construct $\mathcal{D}_{+}$to consist of all data vectors obtained by quantizing $\mathbf{y}_{\mathcal{I}}(\phi)$ for all possible angles $\phi$. Equivalently, $\mathcal{D}_{+}$consists of all data vectors whose $Z F$ decision regions (i.e., the decision regions of the $Z F$ detector) are pierced by the intersection set $\mathcal{L} \cap \mathcal{H}$.

Let $v_{M_{\mathrm{T}}, m}=a_{m} e^{j \phi_{m}}$ denote the $m$ th component of $\mathbf{v}_{M_{\mathrm{T}}}$, and let $b_{m}$ denote the $m$ th component of $\mathbf{y}_{\mathbf{v}_{M_{\mathrm{T}}}}^{\perp}$. Then the $m$ th component of $\mathbf{y}_{\mathcal{I}}(\phi)=\tilde{R} e^{j \phi} \mathbf{v}_{M_{\mathrm{T}}}+\mathbf{y}_{\mathbf{v}_{M_{\mathrm{T}}}}^{\perp}$ can be written as

$$
y_{\mathcal{I}, m}(\phi)=\tilde{R} a_{m} e^{j\left(\phi+\phi_{m}\right)}+b_{m}, \quad m=1, \ldots, M_{\mathrm{T}} .
$$

For $\phi \in[0,2 \pi)$, this represents a circle $\mathcal{C}_{m}$ with radius $\tilde{R} a_{m}$ and center point $b_{m}$ in the symbol plane. When we move along $\mathcal{I}$ (by varying $\phi$ ), all components $y_{\mathcal{I}, m}(\phi)$ of $\mathbf{y}_{\mathcal{I}}(\phi)$ move along their associated circles $\mathcal{C}_{m}$ (see Fig. 3). If one of the $M_{\mathrm{T}}$ components of $\mathbf{y}_{\mathcal{I}}(\phi)$ crosses a boundary line of the symbol quantization regions, $\mathbf{y}_{\mathcal{I}}(\phi)$ moves into a neighboring $\mathrm{ZF}$ decision region. Hereafter, $\phi_{m}^{p}$ will denote the angle $\phi$ for which $\mathcal{C}_{m}$ intersects the $p$ th boundary line.

It follows that the number of intersections of the $M_{\mathrm{T}}$ circles $\mathcal{C}_{m}$ with the $P$ boundary lines of the line-structured symbol constellation equals the number of $\mathrm{ZF}$ decision regions that are pierced by the intersection circle $\mathcal{I}$, which equals the number of data vectors in $\mathcal{D}_{+}$. (Here, we have assumed that at least one circle $\mathcal{C}_{m}$ intersects one boundary line. If no such intersection exists although $\mathcal{L}$ intersects $\mathcal{H}$, we refer to the discussion of Case 2 in Section III-C.) Thus, an upper bound on the number of data vectors in $\mathcal{D}_{+}$is $\left|\mathcal{D}_{+}\right|_{\max }=2 M_{\mathrm{T}} P$ (each one of the $M_{\mathrm{T}}$ component circles $\mathcal{C}_{m}$ has 0 or 2 intersections with each one of the $P$ boundary lines). Fig. 3 shows the circles $\mathcal{C}_{m}$ for a $(4,4)$ channel and 4-QAM modulation. In this example, $M_{\mathrm{T}}=4, P=2,\left|\mathcal{D}_{+}\right|=6$, and $\left|\mathcal{D}_{+}\right|_{\max }=16$.

For efficient calculation of the data vectors $\mathbf{d}^{(i)} \in \mathcal{D}_{+}$, we first determine all "intersection angles" $\phi_{m}^{p}$ and sort them such that they increase monotonically. A first data vector is chosen as $\mathbf{d}^{(1)}=Q\left\{\mathbf{y}_{\mathcal{I}}(\phi)\right\}$ for $\phi=0$. The remaining data vectors $\mathbf{d}^{(2)}, \mathbf{d}^{(3)}, \cdots \in \mathcal{D}_{+}$are then constructed by means 
of the following recursive algorithm. Assume that we just obtained $\mathbf{d}^{(i)} \in \mathcal{D}_{+}$and wish to determine $\mathbf{d}^{(i+1)} \in \mathcal{D}_{+}$that corresponds to the adjacent $\mathrm{ZF}$ decision region reached by $\mathbf{y}_{\mathcal{I}}(\phi)$ when $\phi$ is increased beyond the corresponding intersection angle $\phi_{m}^{p}$. Moving into this neighboring ZF decision region corresponds to updating $\mathbf{d}^{(i)}$ to $\mathbf{d}^{(i+1)}=\mathbf{d}^{(i)}+\Delta \mathbf{d}^{(i)}$. Here, the vector $\Delta \mathbf{d}^{(i)}$ is zero except for the $m$ th component, denoted $\Delta d_{m}^{(i)}$, that depends on $\left(\mathbf{d}^{(i)}\right)_{m}$ and on the $p$ th boundary. For example, for 4-QAM we have (cf. Fig. 3)

$$
\Delta d_{m}^{(i)}= \begin{cases}-2 j \operatorname{Im}\left\{\left(\mathbf{d}^{(i)}\right)_{m}\right\}, & \text { if } p=1 \\ -2 \operatorname{Re}\left\{\left(\mathbf{d}^{(i)}\right)_{m}\right\}, & \text { if } p=2,\end{cases}
$$

that is, the update simply amounts to flipping the imaginary part (for $p=1$ ) or the real part (for $p=2$ ) of $\left(\mathbf{d}^{(i)}\right)_{m}$.

This recursive data vector calculation leads to a recursive calculation of the distances $\psi_{i}=\left\|\mathbf{r}-\mathbf{H d}^{(i)}\right\|$ to be minimized. The distances are updated as $\psi_{i+1}^{2}=\psi_{i}^{2}+\Delta_{i}$, with

$$
\Delta_{i}=\left|\Delta d_{m}^{(i)}\right|^{2}\left\|\mathbf{h}_{m}\right\|^{2}-2 \operatorname{Re}\left\{\Delta d_{m}^{(i)}\left(\mathbf{r}-\mathbf{H d}^{(i)}\right)^{H} \mathbf{h}_{m}\right\},
$$

where $\mathbf{h}_{m}$ denotes the $m$ th column of the channel matrix $\mathbf{H}$. The term $\left(\mathbf{r}-\mathbf{H d}^{(i)}\right)^{H}$ can be calculated recursively, too, and the squared norms $\left\|\mathbf{h}_{m}\right\|^{2}$ can be precalculated.

\section{Efficient Calculation of $\mathcal{D}_{+}$and $\psi_{i}^{2}$ : Case 2}

If $\mathcal{L}$ and $\mathcal{H}$ do not intersect (i.e., $R^{2} \leq\left\|\mathbf{y}_{\mathbf{v}_{M_{\mathrm{T}}}}^{\perp}\right\|^{2}$ ), then $\mathbf{y}_{\mathbf{v}_{M_{\mathrm{T}}}}^{\perp}$ can be shown [1] to be the point of $\mathcal{L}$ closest to $\mathcal{H}$. We thus take $\mathbf{d}^{(1)}=Q\left\{\mathbf{y}_{\mathbf{v}_{M_{\mathrm{T}}}}^{\perp}\right\}$ as the first data vector in $\mathcal{D}_{+}$. Simulation results indicate that it is advantageous to include also the nearest neighbors of $\mathbf{d}^{(1)}$ in $\mathcal{D}_{+}$. These data vectors can easily be found by substituting in $\mathbf{d}^{(1)}$ the nearest-neighbor symbols for the individual components of $\mathbf{d}^{(1)}$. For PSK constellations, we obtain 2 nearest neighbors for each data vector component, yielding $\left|\mathcal{D}_{+}\right|=2 M_{\mathrm{T}}+1$. The distances $\psi_{i}^{2}=\left\|\mathbf{r}-\mathbf{H d}^{(i)}\right\|^{2}$ can again be calculated recursively.

The same procedure is used if $\mathcal{L}$ and $\mathcal{H}$ intersect but no component circle $\mathcal{C}_{m}$ intersects a boundary line.

\section{Summary of the SPA}

The principal steps of the SPA can now be summarized as follows:

- calculation of $\left(\mathbf{H}^{H} \mathbf{H}\right)^{-1}, \mathbf{y}_{\mathrm{ZF}}=\left(\mathbf{H}^{H} \mathbf{H}\right)^{-1} \mathbf{H}^{H} \mathbf{r}$, the dominant eigenvector $\mathbf{v}_{M_{\mathrm{T}}}$ of $\left(\mathbf{H}^{H} \mathbf{H}\right)^{-1}$, and $\hat{\mathbf{d}}_{\text {sub }}$;

- determination of $\mathcal{D}_{+}$and calculation of the corresponding distances $\psi_{i}^{2}$ (see Sections III-B and III-C);

- calculation of the distance for $\hat{\mathbf{d}}_{\text {sub }}$; determination of the minimum distance.

Here, $\hat{\mathbf{d}}_{\text {sub }}$ may be the result of ZF detection, MMSE detection, ZF-based NC detection, or MMSE-based NC detection; the resulting SPA variants will be referred to as ZF/SP, MMSE/SP, NC-ZF/SP, and NC-MMSE/SP detection, respectively. For MMSE/SP and NC-MMSE/SP detection, the reference line $\mathcal{L}$ in (3) is replaced by an "MMSE-based" reference line $\mathcal{L}^{\prime}$ that is still parallel to $\mathbf{v}_{M_{\mathrm{T}}}$ but whose offset is the component of $\mathbf{y}_{\text {MMSE }}$ (rather than $\mathbf{y}_{\mathrm{ZF}}$ ) perpendicular to $\mathbf{v}_{M_{\mathrm{T}}}$.
Thus, MMSE/SP and NC-MMSE/SP detection are obtained simply by replacing in the $\mathrm{ZF} / \mathrm{SP}$ and NC-ZF/SP method, respectively, $\left(\mathbf{H}^{H} \mathbf{H}\right)^{-1}$ with $\left(\mathbf{H}^{H} \mathbf{H}+\frac{\sigma_{w}^{2}}{\sigma_{d}^{2}} \mathbf{I}\right)^{-1}$. The rationale for using $\mathcal{L}^{\prime}$ is that on average $\mathbf{y}_{\mathrm{MMSE}}$ will be closer to the transmitted data vector $\mathbf{d}$ than $\mathbf{y}_{\mathrm{ZF}}$.

\section{E. Computational Complexity}

We will briefly analyze the computational complexity of the SPA add-on (without the calculation of $\hat{\mathbf{d}}_{\text {sub }}$ ) for $M_{\mathrm{T}}=M_{\mathrm{R}}$. The preparatory operations that have to be performed only once for an entire data block during which the channel is constant have a complexity of $\mathcal{O}\left(M_{\mathrm{T}}^{3}\right)$ (due to the calculation of $\left(\mathbf{H}^{H} \mathbf{H}\right)^{-1} \mathbf{H}^{H}$ or $\left.\left(\mathbf{H}^{H} \mathbf{H}+\frac{\sigma_{w}^{2}}{\sigma_{d}^{2}} \mathbf{I}\right)^{-1} \mathbf{H}^{H}\right)$. The complexity of the operations that have to be performed once for each data vector is $\mathcal{O}\left(M_{\mathrm{T}}^{2} P\right)$ (due to the calculation of at most $2 M_{\mathrm{T}} P-1$ efficient distance updates). Note that calculation of the dominant eigenvector $\mathbf{v}_{M_{\mathrm{T}}}$ has complexity $\mathcal{O}\left(M_{\mathrm{T}}^{2}\right)$ if the power method [7] is used.

\section{Simulation Results}

We will now assess the symbol-error rate (SER) performance and computational complexity of the SPA by means of simulation results. In our simulations, we used 4-QAM modulation and MIMO channels with iid Gaussian entries with unit variance. The dominant eigenvector $\mathbf{v}_{M_{\mathrm{T}}}$ was computed by means of the power method [7] using 4 iterations.

\section{A. SER Performance}

Figs. 4(a) and (b) show the SER versus SNR performance ${ }^{1}$ of the various SPA and standard detectors for a $(4,4)$ and $(5,5)$ channel, respectively. The following conclusions can be drawn from these results.

- All SPA detectors perform substantially better than the standard suboptimal detectors. Even the simplest SPA detector (ZF/SP) outperforms the best standard suboptimal detector (NC-MMSE with layer ordering [2][9]).

- All SPA detectors achieve higher diversity orders than the standard suboptimal detectors.

- For the $(4,4)$ channel, the MMSE/SP and NC-MMSE/SP detectors perform as well as the ML detector (the corresponding SER curves in Fig. 4(a) are indistinguishable), and the $\mathrm{ZF} / \mathrm{SP}$ detector achieves near-ML performance.

- For the $(6,6)$ channel, the NC-MMSE/SP detector achieves near-ML performance, and the performance of the other SPA detectors is intermediate between that of the ML detector and that of the best standard suboptimal detector (NC-MMSE with layer ordering).

\section{B. Computational Complexity}

A rough picture of the computational complexity of the various detectors is provided in Table I for three different channels $((4,4),(6,6)$, and $(8,8))$ and 4-QAM modulation. The kflop estimates displayed in Table I were determined

\footnotetext{
${ }^{1}$ The SNR is defined as $\mathrm{E}\left\{\|\mathbf{H d}\|^{2}\right\} / \mathrm{E}\left\{\|\mathbf{w}\|^{2}\right\}=M_{\mathrm{T}} \sigma_{d}^{2} / \sigma_{w}^{2}$.
} 


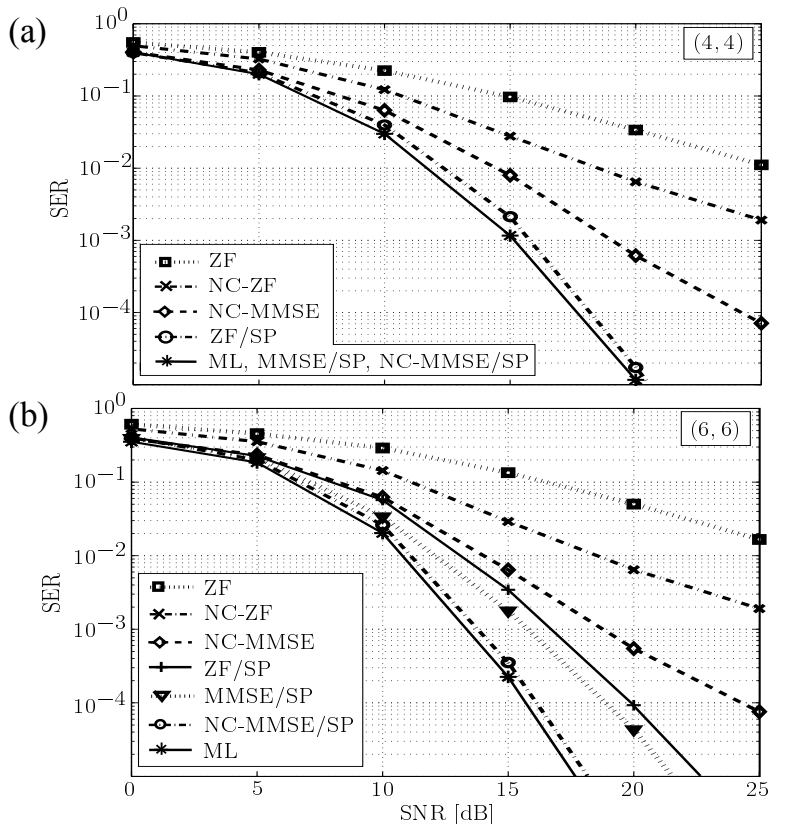

Fig. 4. SER vs. SNR performance of various SPA and standard detectors using 4-QAM modulation: (a) $(4,4)$ system, (b) $(6,6)$ system.

TABLE I

MEASURED COMPLEXITY IN KFLOPS FOR (a) OPERATIONS PERFORMED ONCE PER DATA BLOCK AND (b) OPERATIONS PERFORMED ONCE PER DATA VECTOR.

(a)

\begin{tabular}{|l||c|c|c|c|c|}
\hline \multicolumn{5}{|c|}{ measured kflops - block operations } \\
\hline \hline channel & FPSD & lin. & NC & lin./SP & NC/SP \\
\hline \hline$(4,4)$ & 2.1 & 2.1 & 4.2 & 2.8 & 4.9 \\
\hline$(6,6)$ & 6.4 & 6.4 & 16.2 & 7.8 & 17.6 \\
\hline$(8,8)$ & 14.2 & 14.2 & 45.1 & 16.8 & 47.6 \\
\hline
\end{tabular}

(b)

\begin{tabular}{|l||c|c|c|c|c|c|c|c|}
\hline \multicolumn{8}{|c|}{ measured kflops - vector operations } \\
\hline \hline \multirow{2}{*}{ channel } & \multicolumn{2}{|c|}{ FPSD } & \multirow{2}{*}{ lin. } & \multirow{2}{*}{ NC } & \multicolumn{2}{|c|}{ lin./SP } & \multicolumn{2}{|c|}{ NC/SP } \\
\cline { 2 - 6 } & av. & max. & & & av. & max. & av. & max. \\
\hline \hline$(4,4)$ & 32 & 82 & 0.18 & 0.4 & 1.3 & 2.4 & 1.8 & 2.8 \\
\hline$(6,6)$ & 68 & 300 & 0.37 & 1.1 & 2.5 & 4.7 & 3.6 & 5.7 \\
\hline$(8,8)$ & 122 & 671 & 0.62 & 1.9 & 3.7 & 7.6 & 5.6 & 9.5 \\
\hline
\end{tabular}

using MATLAB V5.3. Table I(a) shows the complexity of the operations that have to be performed once for an entire data block during which the channel is constant (e.g., calculation of $\mathbf{H}^{\#}$ and $\mathbf{v}_{M_{\mathrm{T}}}$ ). Table I(b) shows the complexity of the operations that have to be performed once for each data vector (e.g., determination of $\mathcal{D}_{+}$and $\psi_{i}^{2}$ ). Each table presents only one value for both linear detectors (ZF and MMSE; denoted as "lin.") and one value for both linear detectors combined with the SPA (ZF/SP and MMSE/SP; denoted as "lin./SP"), because there is virtually no difference in complexity.

The ML detector was implemented by means of Fincke and Phost's sphere-decoding (FPSD) algorithm [4]. The computational complexity of FPSD strongly depends on the actual channel realization and SNR. Therefore, in addition to the average complexity, Table I(b) shows the maximum complexity obtained during 10000 simulation runs at an SNR of $10 \mathrm{~dB}$.
The maximum complexity of lin./SP and NC/SP in Table I(b) refers to the case where all circles $\mathcal{C}_{m}$ intersect all boundary lines (see Subsection III-B).

From Table I, the following conclusions can be drawn.

- The overall complexity of the SPA detectors is somewhat higher than that of the standard suboptimal detectors but much lower than that of FPSD. In particular, the complexity of the NC-MMSE/SP detector is just a fraction of the average complexity of FPSD, even though the NC-MMSE/SP detector achieves near-ML performance.

- For the lin./SP detectors, the complexity of the computations performed once per data block is lower than for the $\mathrm{NC}$ detectors but slightly higher than for the linear detectors and FPSD (due to the additional calculation of $\mathbf{v}_{M_{\mathrm{T}}}$ ).

- For the lin./SP detectors, the average complexity of the computations performed once per data vector is about twice that of the NC detectors but significantly lower (by a factor of about 25...33) than that of FPSD.

\section{Summary And CONCLusions}

We developed the sphere-projection algorithm (SPA) that is a computationally efficient add-on to standard suboptimal detectors for MIMO spatial multiplexing systems. The SPA was motivated by the observation that the poor performance of standard suboptimal detectors compared to maximum likelihood (ML) detection is primarily caused by the occurrence of "bad" (i.e., poorly conditioned) channel realizations. The SPA detectors are robust to bad channels and capable of achieving near-ML performance and significantly increased diversity gains at a fraction of the computational complexity of Fincke and Phost's sphere-decoding algorithm for ML detection. The SPA detectors also compare favorably to nulling-andcancelling schemes because they achieve significantly larger diversity gains at a comparable computational complexity.

\section{REFERENCES}

[1] H. Artés, D. Seethaler, and F. Hlawatsch, "Efficient detection algorithms for MIMO channels: A geometrical approach to approximate ML detection," IEEE Trans. Signal Processing, Special Issue on Signal Processing for Multiple-Input Multiple-Output (MIMO) Wireless Communications Systems, vol. 51, 2003, to appear.

[2] G. Golden, C. Foschini, R. Valenzuela, and P. Wolniansky, "Detection algorithm and initial laboratory results using V-BLAST space-time communications architecture," Elect. Lett., vol. 35, pp. 14-16, jan. 1999.

[3] S. M. Kay, Fundamentals of Statistical Signal Processing: Estimation Theory. Englewood Cliffs (NJ): Prentice Hall, 1993.

[4] U. Fincke and M. Phost, "Improved methods for calculating vectors of short length in a lattice, including a complexity analysis," Math. of Comp., vol. 44, pp. 463-471, April 1985.

[5] S. M. Kay, Fundamentals of Statistical Signal Processing: Detection Theory. Upper Saddle River (NJ): Prentice Hall, 1998.

[6] B. Hassibi and H. Vikalo, "On the expected complexity of sphere decoding," in Proc. 35th Asilomar Conf. Signals, Systems, Computers, Pacific Grove (CA), Nov. 2001, pp. 1051-1055.

[7] G. H. Golub and C. F. Van Loan, Matrix Computations, 3rd ed. Baltimore: Johns Hopkins University Press, 1996.

[8] C. W. Therrien, Discrete Random Signals and Statistical Signal Processing. Englewood Cliffs (NJ): Prentice Hall, 1992.

[9] H. Rupp, M. Guillaud, and S. Das, "On MIMO decoding algorithms for UMTS," in Proc. 35th Asilomar Conf. Signals, Systems, Computers, vol. 2, Pacific Grove, CA, Nov. 2001, pp. 975-979. 